\title{
More on Selective Covering Properties in Bitopological Spaces
}

\author{
Ljubiša D. R. Kočinac $\mathbb{D}^{1}$ and Selma Özçă̆ $\mathbb{D}^{2}$ \\ ${ }^{1}$ University of Niš, Faculty of Sciences and Mathematics, 18000 Niš, Serbia \\ ${ }^{2}$ Hacettepe University, Department of Mathematics, Ankara, Turkey \\ Correspondence should be addressed to Ljubiša D. R. Kočinac; lkocinac@gmail.com
}

Received 24 January 2021; Accepted 31 March 2021; Published 14 April 2021

Academic Editor: Frédéric Mynard

Copyright (C) 2021 Ljubiša D. R. Kočinac and Selma Özçağ. This is an open access article distributed under the Creative Commons Attribution License, which permits unrestricted use, distribution, and reproduction in any medium, provided the original work is properly cited.

\begin{abstract}
In this study, we continue our investigation of selective covering properties in bitopological spaces. We discuss their behaviour under certain kinds of mappings. We also introduce selective versions of the ccc property and the star-ccc property in bitopological spaces and give few of their relations with other selective properties. Also, we consider preservation of selective covering properties of bitopological spaces under some known relations in bitopological context.
\end{abstract}

\section{Introduction}

Throughout the study, we use the standard topological notation and terminology, mainly as in $[1]$. By $\mathbb{N}$ and $\mathbb{R}$, we denote the set of natural numbers and the set of real numbers, respectively. By $X$ or $(X, \tau)$, we denote a topological space, while $\left(X, \tau_{1}, \tau_{2}\right)$ denotes a bitopological space [2] (called also bispace), that is a set $X$ equipped with two (in general, unrelated) topologies. The closure and interior of a subset $A$ of a space $(X, \tau)$ are denoted by $\mathrm{Cl}(A)$ and $\operatorname{Int}(A)$, respectively. When $\left(X, \tau_{1}, \tau_{2}\right)$ is a bitopological space and $A \subset X$, then $\mathrm{Cl}_{\tau_{i}}(A)$ and $\operatorname{Int}_{\tau_{i}}(A), i=1,2$, denote the closure and interior in the space $\left(X, \tau_{i}\right)$.

If $A$ is a subset of a topological space $X, x \in X$, and $\mathscr{F}$ is a family of subsets of $X$, then $\operatorname{St}(A, \mathscr{F})=\bigcup\{F \in \mathscr{F}$ : $A \cap F \neq \varnothing\}$, and $\operatorname{St}(x, \mathscr{F})=\operatorname{St}(\{x\}, \mathscr{F})$ denote the star of $A$ and $x$ with respect to $\mathscr{F}$.

For a space $(X, \tau)$, we also use the following notations:

(i) $\mathcal{O}$ is the collection of all open covers of $X$

(ii) $\mathcal{O}_{D}=\{\mathscr{U} \subset \tau: \cup \mathscr{U}$ is dense in $X\} \quad(\mathscr{U}$ with this property is called an open weak cover of $X$ )

(iii) $\overline{\mathcal{O}}=\{\mathscr{U} \subset \tau: \cup\{\bar{U}: U \in \mathscr{U}\}=X\} \quad(\mathscr{U}$ with this property is called an open almost cover of $X$ )

In case of bitopological spaces, we write $\mathcal{O}^{(i)}, \mathcal{O}_{D}^{(i)}, \overline{\mathcal{O}}^{(i)}$ to indicate that $\mathcal{O}, \mathscr{O}_{D}, \overline{\mathcal{O}}$ is related to the space $\left(X, \tau_{i}\right), i=1,2$.
If $\mathscr{A}$ and $\mathscr{B}$ are families of sets, then

(1) The symbol $S_{\text {fin }}(\mathscr{A}, \mathscr{B})$ denotes the selection hypothesis: for each sequence $\left(A_{1}, A_{2}, \ldots\right)$ of elements of $\mathscr{A}$, there is a sequence $\left(B_{1}, B_{2}, \ldots\right)$, such that for each $n \in \mathbb{N}, B_{n}$ is a finite subset of $A_{n}$, and $\cup_{n \in \mathbb{N}} B_{n} \in \mathscr{B}$ (see [3]);

(2) The symbol $S_{1}(\mathscr{A}, \mathscr{B})$ denotes the selection hypothesis: for each sequence $\left(A_{1}, A_{2}, \ldots\right)$ of elements of $\mathscr{A}$, there is a sequence $\left(b_{1}, b_{2}, \ldots\right)$, such that $b_{n} \in A_{n}$ for each $n \in \mathbb{N}$, and $\left\{b_{n}: n \in \mathbb{N}\right\} \in \mathscr{B}$ (see [3]).

The following covering properties are defined in this way:

(i) $S_{\text {fin }}(\mathcal{O}, \mathcal{O})$ is the Menger property [4]

(ii) $S_{1}(\mathcal{O}, \mathcal{O})$ is the Rothberger property [5]

(iii) $S_{\text {fin }}\left(\mathcal{O}, \mathcal{O}_{D}\right)$ is the weak Menger property [6] (see also $[7,8])$

(iv) $S_{\text {fin }}(\mathcal{O}, \overline{\mathcal{O}})$ is the almost Menger property [9] (see also $[10])$

(v) $S_{1}\left(\mathcal{O}, \mathcal{O}_{D}\right)$ is the weak Rothberger property [6]

(vi) $S_{1}(\mathcal{O}, \overline{\mathcal{O}})$ is the almost Rothberger property [9]

A space $X$ has the Hurewicz property if for each sequence $\left(\mathscr{U}_{1}, \mathscr{U}_{2}, \ldots\right)$ of open covers of $X$, there is a sequence 
$\left(\mathscr{V}_{1}, \mathscr{V}_{2}, \ldots\right)$, such that for each $n \in \mathbb{N}, \mathscr{V}_{n}$ is a finite subset of $\mathscr{U}_{n}$, and each $x \in X$ belongs to $\bigcup \mathscr{V}_{n}$ for all but finitely many $n[11]$.

For selection principles theory see [12].

Recall that a family of pairwise disjoint open sets in a topological space $X$ is called a cellular open family. $X$ is said to be a ccc space if every cellular open family in it is countable.

Observe that any separable space, clearly, is ccc.

Selective version of the ccc space known as "selectively ccc spaces" was first introduced (under this name) by Aurichi [13].

Definition 1. (see [13]) Let $(X, \tau)$ be a topological space. $X$ is selectively ccc if for every sequence $\left(\mathscr{A}_{n}: n \in \mathbb{N}\right)$ of maximal cellular open families in $X$, there is a sequence $\left(A_{n}: n \in \mathbb{N}\right)$, such that for each $n, A_{n} \in \mathscr{A}_{n}$ and $\cup_{n \in \mathbb{N}} A_{n}$ is dense in $X$.

In fact, these spaces were considered in the literature under different names. In $[14,15]$, Scheepers (first) studied the selectively ccc spaces under the notation $S_{1}(\mathscr{D}, \mathscr{D})$, where $\mathscr{D}$ is the collection of open families with a dense union (i.e., $\mathscr{D}$ is $\mathscr{O}_{D}$ in our notation). In [16], these spaces have been studied under the notation $S_{1}\left(\mathscr{D}_{O}, \mathscr{D}\right)$, where $\mathscr{D}_{O}$ denotes the collection of open families with a dense union. In both studies $[13,16]$, it was explained that all these three definitions are equivalent.

In this study, we consider selectively ccc spaces as spaces belonging to the class $S_{1}\left(\mathscr{O}_{D}, \mathcal{O}_{D}\right)$.

If $\mathscr{A}$ and $\mathscr{B}$ are collections of covers of a space $X$, then

(1) The symbol $S S_{1}(\mathscr{A}, \mathscr{B})$ denotes the selection hypotheses that for each sequence $\left(\mathscr{U}_{1}, \mathscr{U}_{2}, \ldots\right)$ of elements of $\mathscr{A}$, there is a sequence $\left(x_{1}, x_{2}, \ldots\right)$ of elements of $X$ such that $\left\{\operatorname{St}\left(x_{n}, \mathscr{U}_{n}\right): n \in \mathbb{N}\right\} \in \mathscr{B}$ (see [17])

(2) The symbol $S S_{\mathscr{O}, 1}^{*}(\mathscr{A}, \mathscr{B})$ denotes the selection hypotheses: for each open cover $\mathscr{U}$ of $X$ and each sequence $\left(\mathscr{U}_{1}, \mathscr{U}_{2}, \ldots\right)$ of elements of $\mathscr{A}$, there is a sequence $\left(U_{1}, U_{2}, \ldots\right)$, such that for each $n, U_{n} \in \mathscr{U}_{n}$ and $\operatorname{St}\left(\cup_{n \in \mathbb{N}} U_{n}, \mathscr{U}\right) \in \mathscr{B}$ (see [18])

In [19], the following spaces have been defined and studied.

Definition 2. (see [19]) A topological space $X$ is said to be selectively star-ccc if it belongs to the class $S S_{\mathscr{O}, 1}^{*}\left(\mathcal{O}_{D}, \mathcal{O}\right)$.

In this study, we introduce and study the selective versions of the ccc property and selectively star-ccc property in bitopological spaces. We establish some relations with other selective bitopological properties.

The study is organized as follows. In Section 2, we consider preservation of the bitopological almost Menger and almost Rothberger properties under certain classes of mappings. Section 3 deals with selectively ccc and selectively star-ccc bitopological spaces. Finally, in Section 4, we discuss the behaviour of several topological and bitopological covering properties under known relations between the two topologies.

\section{Mappings and Almost Menger Bispaces}

The first study of weak covering properties in the bitopological context began with the study [20] on the almost Menger property in bitopological spaces and continued in $[21,22]$ where some results on almost Menger and weak Menger properties in the bitopological spaces were obtained. Selective properties in bitopological spaces have been studied in [23-25].

Let us recall the following definition.

Definition 3. (see [20]). A bitopological space $\left(X, \tau_{1}, \tau_{2}\right)$ is said to be $(i, j)$-almost Menger, $i, j=1,2$, if for each sequence $\left(\mathcal{U}_{n}: n \in \mathbb{N}\right)$ of $\tau_{i}$-open covers of $X$, there exists a sequence $\left(\mathscr{V}_{n}: n \in \mathbb{N}\right)$ of finite families, such that for each $n$, $\mathscr{V}_{n} \subset \mathscr{U}_{n}$ and $X=\cup_{n \in \mathbb{N}} \cup_{V \in \mathscr{V}}{ }_{n} \mathrm{Cl}_{\tau_{j}}(V)$.

This section focuses on the behaviour of almost Menger, almost Rothberger, and almost Hurewicz properties under some kinds of mappings between bitopological spaces.

We recall the following definitions. A mapping $f:\left(X, \tau_{1}, \tau_{2}\right) \longrightarrow\left(Y, \sigma_{1}, \sigma_{2}\right)$ is called

(1) $(i, j)-\theta$-continuous [26] (respectively, $(i, j)$-strongly $\theta$-continuous [27]) if for each $x \in X$ and each $\sigma_{i}$-open set $V \subset Y$ containing $f(x)$, there exists a $\tau_{i}$-open set $U \subset X$ containing $x$, such that $f\left(\mathrm{Cl}_{\tau_{j}}(U)\right) \subset \mathrm{Cl}_{\sigma_{j}}(V)$ (respectively, $\left.f\left(\mathrm{Cl}_{\tau_{j}}(U)\right) \subset V\right)$. It is obvious that each $(i, j)$-strongly $\theta$-continuous mapping is $(i, j)-\theta$-continuous.

(2) $(i, j)$-almost continuous [28] if $f^{\leftarrow}(B)$ is the $\tau_{i}$-open set in $X$ for every $(i, j)$-regular open set $B$ in $Y$. In addition, $f$ is called $p$-almost continuous if it is $(1,2)$ and $(2,1)$-almost continuous. Recall that a set $B$ in a bitopological space $\left(X, \tau_{1}, \tau_{2}\right)$ is $(i, j)$-regular open [29] if $B=\operatorname{Int}_{\tau_{i}} \mathrm{Cl}_{\tau_{j}}(B)$.

We note that $p$-almost continuous mappings are $(i, j)-\theta$-continuous [30], so the following result generalizes the result in [20], which states that the $(i, j)$-almost Menger property is preserved by $p$-almost continuous mappings.

Theorem 1 (see [21]). An $(i, j)-\theta$-continuous image of an $(i, j)$-almost Menger bitopological space is $(i, j)$-almost Menger.

In [21], the definitions of $(i, j)$-almost $\gamma$-sets and the $(i, j)$-almost Hurewicz property were introduced, and the following theorem was obtained.

Theorem 2 (see [21]). Let $\left(X, \tau_{1}, \tau_{2}\right)$ be an $(i, j)$-almost $\gamma$-set and $\left(Y, \sigma_{1}, \sigma_{2}\right)$ be a bitopological space. If $f: X \longrightarrow Y$ is an $(i, j)$ - $\theta$-continuous surjection, then $\left(Y, \sigma_{1}, \sigma_{2}\right)$ is $(i, j)$-almost Hurewicz.

Now, we consider the behaviour of the $(i, j)$-almost Rothberger property under $(i, j)-\theta$-continuous mappings.

A bitopological space $\left(X, \tau_{1}, \tau_{2}\right)$ is said to be $(i, j)$-almost Rothberger, $(i, j=1,2)$, if for each sequence 
$\left(\mathcal{U}_{n}: n \in \mathbb{N}\right)$ of $\tau_{i}$-open covers of $X$, there is a sequence $\left(U_{n}: n \in \mathbb{N}\right)$, such that for each $n, U_{n} \in \mathcal{U}_{n}$ and $X=\cup_{n \in \mathbb{N}} \mathrm{Cl}_{\tau_{j}}\left(U_{n}\right)[20]$.

Theorem 3. An $(i, j)$ - $\theta$-continuous image of an $(i, j)$-almost Rothberger bitopological space is $(i, j)$-almost Rothberger.

Proof. Let $\left(X, \tau_{1}, \tau_{2}\right)$ be an $(i, j)$-almost Rothberger bitopological space. Let $Y=f(X)$ be the image of $X$, where $f:\left(X, \tau_{1}, \tau_{2}\right) \longrightarrow\left(Y, \sigma_{1}, \sigma_{2}\right)$ is an $(i, j)$ - $\theta$-continuous mapping.

Let $\left(\mathscr{V}_{n}: n \in \mathbb{N}\right)$ be a sequence of $\sigma_{i}$-open covers of $Y$. For each $n \in \mathbb{N}$ and fixed $x \in X$, there is a set $V_{x, n} \in \mathscr{V}_{n}$, such that $f(x) \in V_{x, n}$. Since $f$ is $(i, j)$ - $\theta$-continuous, there exists a $\tau_{i}$-open set $U_{x, n} \subset X$ containing $x$, such that $f\left(\mathrm{Cl}_{\tau_{j}}\left(U_{x, n}\right)\right) \subset \mathrm{Cl}_{\sigma_{j}}\left(V_{x, n}\right)$. For each $n, \mathscr{U}_{n}:=\left\{U_{x, n}\right.$ : $x \in X\}$ is a $\tau_{i}$-open cover of $X$. Apply the fact that $X$ is $(i, j)$-almost Rothberger. There is a sequence $\left(x_{n}: n \in \mathbb{N}\right)$, such that for each $n \in \mathbb{N}, U_{x_{n}, n} \in \mathcal{U}_{n}$ and $X=\cup_{n \in \mathbb{N}} \mathrm{Cl}_{\tau_{j}}\left(U_{x_{n}, n}\right)$. For each $n$ and $U_{x_{n}, n}$, we assign a set $V_{x_{n}, n} \in \mathscr{V}_{n}$, such that $f\left(\mathrm{Cl}_{\tau_{j}}\left(U_{x_{n}, n}\right)\right) \subset \mathrm{Cl}_{\sigma_{j}}\left(V_{x_{n}, n}\right)$. We obtain the sequence $\left(V_{x_{n}, n}: n \in \mathbb{N}\right)$, with $V_{x_{n}, n} \in \mathscr{V}_{n}$ and

$$
\begin{aligned}
Y & =f(X)=f\left(\underset{n \in \mathbb{N}}{\cup} \mathrm{Cl}_{\tau_{j}}\left(U_{x_{n}, n}\right)\right) \\
& =\underset{n \in \mathbb{N}}{\cup} f\left(\mathrm{Cl}_{\tau_{j}}\left(U_{x_{n}, n}\right)\right) \subset \underset{n \in \mathbb{N}}{\cup} \mathrm{Cl}_{\sigma_{j}}\left(V_{x_{n}, n}\right) .
\end{aligned}
$$

This means that $\left(Y, \sigma_{1}, \sigma_{2}\right)$ is $(i, j)$-almost Rothberger.

Corollary 1. The $(i, j)$-almost Rothberger property is preserved by p-almost continuous mappings.

Theorem 4. If $f:\left(X, \tau_{1}, \tau_{2}\right) \longrightarrow\left(Y, \sigma_{1}, \sigma_{1}\right)$ is an $(i, j)$-strongly $\theta$-continuous mapping and $X$ is $(i, j)$-almost Menger, then $\left(Y, \sigma_{i}\right)$ is Menger.

Proof. Let $\left(\mathscr{V}_{n}: n \in \mathbb{N}\right)$ be a sequence of $\sigma_{i}$-open covers of $Y$ and $x \in X$. For each $n \in \mathbb{N}$, there exists a set $V_{x, n} \in \mathscr{V}_{n}$ with $f(x) \in V_{x, n}$. Since $f$ is $(i, j)$-strongly $\theta$-continuous, there is a $\tau_{i}$-open set $U_{x, n} \subset X$ with $x \in U_{x, n}$, such that $f\left(\mathrm{Cl}_{\tau_{j}}\left(U_{x, n}\right)\right) \subset V_{x, n}$. Then, $\mathscr{U}_{n}:=\left\{U_{x, n}: x \in X\right\}$ is a $\tau_{i}$-open cover of $X$. Since $X$ is $(i, j)$-almost Menger bitopological space, there exists a sequence $\left(\mathscr{H}_{n}: n \in \mathbb{N}\right)$, such that for each $n \in \mathbb{N}, \mathscr{H}_{n}$ is a finite subset of $\mathscr{U}_{n}$, and $X=\cup_{n \in \mathbb{N}} \cup\left\{\mathrm{Cl}_{\tau_{j}}(H): H \in \mathscr{H}_{n}\right\}$. Now, for each $n \in \mathbb{N}$ and $H \in \mathscr{H}_{n}$, we can choose $V_{H} \in \mathscr{V}_{n}$, such that $f\left(\mathrm{Cl}_{\tau_{j}}(H)\right) \subseteq V_{H}$. Let $\mathscr{F}_{n}=\left\{V_{H}: H \in \mathscr{H}_{n}\right\}$; we obtain a sequence $\left(\mathscr{F}_{n}: n \in \mathbb{N}\right)$ of finite subsets of $\mathscr{V}_{n}, n \in \mathbb{N}$. We will show $\bigcup \mathscr{F}_{n}$ is a $\sigma_{i}$-cover of $Y$. Indeed,

$$
\begin{aligned}
Y= & f(X)=f\left(\cup_{n \in \mathbb{N}} \bigcup\left\{\mathrm{Cl}_{\tau_{j}}(H): H \in \mathscr{H}_{n}\right\}\right), \\
= & \cup_{n \in \mathbb{N}} \bigcup\left\{f\left(\mathrm{Cl}_{\tau_{j}}(H)\right): H \in \mathscr{H}_{n}\right\}, \\
& \subset \cup_{n \in \mathbb{N}} \bigcup\left\{V_{H}: H \in \mathscr{H}_{n}\right\} .
\end{aligned}
$$

This shows that $\left(Y, \sigma_{i}\right)$ is Menger.

Remark 1. It is obvious that if $f:\left(X, \tau_{1}, \tau_{2}\right) \longrightarrow\left(Y, \sigma_{1}, \sigma_{2}\right)$ is an $(i, j)$-strongly $\theta$-continuous mapping, then $f$ is $i$-continuous; furthermore, if $\left(X, \tau_{1}, \tau_{2}\right)$ is the $(i, j)$-regular bitopological space, then the reverse implication is also true.

Corollary 2. If $f:\left(X, \tau_{1}, \tau_{2}\right) \longrightarrow\left(Y, \sigma_{1}, \sigma_{1}\right)$ is $i$-continuous mapping and $X$ is $(i, j)$-regular, $(i, j)$-almost Menger bitopological space, then $\left(Y, \sigma_{i}\right)$ is Menger.

Now, recall the notions of contra continuous and precontinuous mappings in bitopological spaces. A mapping $f:\left(X, \tau_{1}, \tau_{2}\right) \longrightarrow\left(Y, \sigma_{1}, \sigma_{2}\right)$ is said to be $(i, j)$-contracontinuous and $(i, j)$-precontinuous [29], if the preimage of each $\sigma_{i}$-open set $V$ of $Y$ is $\tau_{j}$-closed in $X$ $\left((i, j)\right.$-preopen in $X$, i.e., $\left.f^{\leftarrow}(V) \subset \operatorname{Int}_{\tau_{i}} \mathrm{Cl}_{\tau_{j}}\left(f^{\leftarrow}(V)\right)\right)$, where $i, j=1,2$.

Theorem 5. If $\left(X, \tau_{1}, \tau_{2}\right)$ is the $(i, j)$ almost Menger bitopological space and $f:\left(X, \tau_{1}, \tau_{2}\right) \longrightarrow\left(Y, \sigma_{1}, \sigma_{1}\right)$ is an $(i, j)$-contracontinuous and $(i, j)$-precontinuous mapping, then $\left(Y, \sigma_{i}\right)$ is a Menger space.

Proof. Let $\left(\mathscr{V}_{n}: n \in \mathbb{N}\right)$ be a sequence of covers of $Y$ by $\sigma_{i}$-open sets. Since $f$ is $(i, j)$-contra continuous, for each $n \in \mathbb{N}$ and $V \in \mathscr{V}_{n}$, the set $f^{\leftarrow}(V)$ is $\tau_{j}$-closed in $X$. Then, we have $f^{\leftarrow}(V) \subset \operatorname{Int}_{\tau_{i}} \mathrm{Cl}_{\tau_{j}}\left(f^{\leftarrow}(V)\right)$, since $f$ is $(i, j)$-precontinuous. Now, we obtain $f^{\leftarrow}(V) \subset \operatorname{Int}_{\tau_{i}}\left(f^{\leftarrow}(V)\right)$, which gives $f^{\leftarrow}(V)=\operatorname{Int}_{\tau_{i}}\left(f^{\leftarrow}(V)\right)$. Then, for each $n \in \mathbb{N}$, we set $\mathcal{U}_{n}:=\left\{f^{\leftarrow}(V): V \in \mathscr{V}_{n}\right\}$. Then, $\left(\mathcal{U}_{n}: n \in \mathbb{N}\right)$ is a sequence of $\tau_{i}$-open covers of $X$. Since $X$ is $(i, j)$-almost Menger, there exists a sequence $\left(\mathscr{H}_{n}: n \in \mathbb{N}\right)$, such that for each $n \in \mathbb{N}, \mathscr{H}_{n}$ is a finite subset of $\mathscr{U}_{n}$ and $\cup_{n \in \mathbb{N}} \cup\left\{\mathrm{Cl}_{\tau_{j}}(H): H \in \mathscr{H}_{n}\right\}=X$. Let $\mathscr{F}_{n}=\left\{f(H): H \in \mathscr{H}_{n}\right\}$. It is obvious that $\left(\mathscr{F}_{n}: n \in \mathbb{N}\right)$ is a sequence of finite subsets of $\mathscr{V}_{n}$ for each $n \in \mathbb{N}$, and $\cup_{n \in \mathbb{N}} \mathscr{F}_{n}$ is an $\sigma_{i}$-open cover of $Y$ which means that $\left(Y, \sigma_{i}\right)$ is a Menger space.

A mapping $f:\left(X, \tau_{1}, \tau_{2}\right) \longrightarrow\left(Y, \sigma_{1}, \sigma_{2}\right)$ is said to be $(i, j)$-weakly continuous [26] if for each $x \in X$ and each $\sigma_{i}$-open subset $V \subset Y$ containing $f(x)$, there exist a $\tau_{i}$-open set $U \subset X$ with $x \in U$, such that $f(U) \subset \mathrm{Cl}_{\sigma_{j}}(V)$.

Theorem 6. If $f:\left(X, \tau_{1}, \tau_{2}\right) \longrightarrow\left(Y, \sigma_{1}, \sigma_{2}\right)$ is an $(i, j)$-weakly continuous mapping and $\left(X, \tau_{i}\right)$ is Menger, then $\left(Y, \sigma_{1}, \sigma_{2}\right)$ is an $(i, j)$-almost Menger space. 
Proof. Let $\left(X, \tau_{i}\right)$ be a Menger space and $\left(\mathscr{V}_{n}: n \in \mathbb{N}\right)$ be a sequence of $\sigma_{i}$-open covers of $Y$. Let $x \in X$, then for each $n \in \mathbb{N}$, there exists $V \in \mathscr{V}_{n}$ with $f(x) \in V$. Since $f$ is $(i, j)$-weakly continuous, there is a $\tau_{i}$-open set $U_{x, n} \subset X$ with $x \in U_{x, n}$ and $f\left(U_{x, n}\right) \subset \mathrm{Cl}_{\sigma_{j}}(V)$. Now, let $\mathscr{U}_{n}:=\left\{U_{x, n}\right.$ : $x \in X\}$. Clearly, $\mathcal{U}_{n}$ is a $\tau_{i}$-open cover of $X$. Since $\left(X, \tau_{i}\right)$ is Menger, we find a sequence $\left(\mathscr{F}_{n}: n \in \mathbb{N}\right)$ of finite sets, such that for each $n \in \mathbb{N}, \mathscr{F}_{n} \subset \mathscr{U}_{n}$ and $\cup_{n \in \mathbb{N}} \mathscr{F}_{n}$ is a $\tau_{i}$-open cover of $X$. For each $n \in \mathbb{N}$ and $U \in \mathscr{F}_{n}$, we can choose a set $V_{U} \in \mathscr{V}_{n}$, such that $f(U) \subset \mathrm{Cl}_{\sigma_{j}}\left(V_{U}\right)$. Set $\mathscr{H}_{n}=\left\{V_{U}\right.$ : $\left.U \in \mathscr{F}_{n}\right\}$. We show that $\bigcup \mathscr{H}_{n}$ is a cover of $Y$. Indeed, as $X=\cup_{n \in \mathbb{N}} \bigcup\left\{U: U \in \mathscr{F}_{n}\right\}$, we have

$$
\begin{aligned}
Y= & f(X)=f\left(\cup_{n \in \mathbb{N}} \bigcup\left\{U: U \in \mathscr{F}_{n}\right\}\right) \\
& \subset \cup_{n \in \mathbb{N}} \bigcup\left(f(U): U \in \mathscr{F}_{n}\right) \\
& \subset \cup_{n \in \mathbb{N}} \bigcup\left\{\mathrm{Cl}_{\sigma_{j}}\left(V_{U}\right): U \in \mathscr{F}_{n}\right\} .
\end{aligned}
$$

So, $\left(Y, \sigma_{1}, \sigma_{2}\right)$ is $(i, j)$-almost Menger.

Remark 2. Since $(i, j)$ - $\theta$-continuous mappings are $(i, j)$-weakly continuous, Theorem 6 is also true under $\theta$-continuous mappings.

\section{Selectively ccc and Selectively Star- ccc Bispaces}

\subsection{Selectively ccc Bispaces}

Definition 4. A bitopological space $\left(X, \tau_{1}, \tau_{2}\right)$ is said to satisfy $i$-countable chain condition (for short $i$-ccc), $i=1,2$, if the topological space $\left(X, \tau_{i}\right)$ satisfies countable chain condition. $X$ satisfies countable chain condition if it satisfies $i$-countable chain condition for $i=1,2$.

Definition 5. A bitopological space $\left(X, \tau_{1}, \tau_{2}\right)$ is $(i, j)$-selectively ccc $(i, j=1,2)$ if for every sequence $\left(\mathcal{U}_{n}: n \in \mathbb{N}\right)$ of elements of $\mathcal{O}_{D}^{(i)}$, there is a sequence $\left(U_{n}: n \in \mathbb{N}\right)$, such that $U_{n} \in \mathcal{U}_{n}, n \in \mathbb{N}$, and $\cup_{n \in \mathbb{N}} U_{n}$ is dense in $\left(X, \tau_{j}\right)$, i.e., if $S_{1}\left(\mathcal{O}_{D}^{(i)}, \mathcal{O}_{D}^{(j)}\right)$ is satisfied.

Proposition 1. Let $\left(X, \tau_{1}, \tau_{2}\right)$ be a bitopological space such that $\tau_{1} \leq \tau_{2}$ (i.e., the topology $\tau_{1}$ is weaker than $\tau_{2}$ ).

(1) If $\left(X, \tau_{1}, \tau_{2}\right)$ is $(1,2)$-selectively ccc, then $\left(X, \tau_{1}\right)$ is selectively ccc

(2) If $\left(X, \tau_{2}\right)$ is selectively ccc, then $\left(X, \tau_{1}, \tau_{2}\right)$ is $(2,1)$-selectively ccc

Since the selectively ccc property is stronger than the ccc property, we have the following.

Corollary 3. Let $\left(X, \tau_{1}, \tau_{2}\right)$ be a bitopological space such that $\tau_{1} \leq \tau_{2}$. If $X$ is $(1,2)$-selectively ccc bispace, then $\left(X, \tau_{1}\right)$ has the ccc property.
Proposition 2. Let $\left(X, \tau_{1}, \tau_{2}\right)$ be a bitopological space and $Y \subset X$ be $\tau_{i}$-closed and $\tau_{j}$-open subspace in $X$. If $X$ is $(i, j)$-selectively ccc, then $\left(Y, \tau_{1}\left|Y, \tau_{2}\right| Y\right)$ is $(i|Y, j| Y)$-selectively ccc.

Proof. (For $i=1$ and $j=2$ ). Let $Y$ be a $\tau_{1}$-closed and $\tau_{2}$-open subset of $X$ and let $\left(\mathscr{U}_{n}: n \in \mathbb{N}\right)$ be a sequence of members of $\mathcal{O}_{D}^{(1 \mid Y)}$. Thus, for each $n \in \mathbb{N}, \mathscr{V}_{n}=\mathscr{U}_{n} \bigcup\{X \backslash Y\}$ is an element of $\mathcal{O}_{D}^{(1)}$. Since $X$ is $(1,2)$-selectively ccc, there is a sequence $\left(V_{n}: n \in \mathbb{N}\right)$, such that for every $n \in \mathbb{N}, V_{n} \in \mathscr{V}_{n}$ and $\mathrm{Cl}_{2}\left(\cup_{n \in \mathbb{N}} V_{n}\right)=X$. If $V_{n} \in \mathcal{U}_{n}$, we set $W_{n}=V_{n}$; if $V_{n}=X \backslash Y$, then we take $W_{n}$ to be any element from $\mathcal{U}_{n}$. Then, it is clear that $\mathrm{Cl}_{2 \mid Y}\left(\cup_{n \in \mathbb{N}} W_{n}\right)=Y$.

Recall that a subset $A$ of a bispace $\left(X, \tau_{1}, \tau_{2}\right)$ is called bidense (here called double dense or shorty $d$-dense) in $X$ if $A$ is dense in both $\left(X, \tau_{1}\right)$ and $\left(X, \tau_{2}\right) . X$ is $d$-separable if there is a countable set $A$ which is $d$-dense in $X$.

Theorem 7. Let $Y \subset X$ be a d-dense set in a bispace $\left(X, \tau_{1}, \tau_{2}\right) . X$ is $(i, j)$-selectively $c c c$ if and only if $Y$ is $(i|Y, j| Y)$ selectively ccc.

Proof. (For $i=1$ and $j=2)$. Let $\left(\mathscr{V}_{n}: n \in \mathbb{N}\right)$ be a sequence of $\tau_{1} \mid Y$-open families such that $\bigcup \mathscr{V}_{n}$ is dense in $\left(Y, \tau_{1} \mid Y\right)$. Since $Y$ is $\tau_{1}$-dense, then each $\bigcup \mathscr{V}_{n}$ is dense in $\left(X, \tau_{1}\right)$. As $X$ is $(1,2)$-selectively ccc, there is a sequence $\left(V_{n}: n \in \mathbb{N}\right)$, such that for each $V_{n} \in \mathscr{V}_{n}, n \in \mathbb{N}$, and $\cup_{n \in \mathbb{N}} V_{n}$ is dense in $\left(X, \tau_{2}\right)$. Since $Y$ is dense in $\left(X, \tau_{2}\right)$, then $\cup_{n \in \mathbb{N}} V_{n}$ is dense in $\left(Y, \tau_{2} \mid Y\right)$.

Let $\left(\mathcal{U}_{n}: n \in \mathbb{N}\right)$ be a sequence of $\tau_{1}$-open families, such that each $\bigcup \mathcal{U}_{n}$ is dense in $\left(X, \tau_{1}\right)$. The restriction to $Y$, $\mathcal{U}_{n} \mid Y=\left\{U \cap Y: U \in \mathcal{U}_{n}\right\}$ is a $\tau_{1} \mid Y$-open family for each $n$. Since $Y$ is $(1|Y, 2| Y)$-selectively ccc, there is a sequence $\left(V_{n}: n \in \mathbb{N}\right)$, such that $V_{n} \in \mathcal{U}_{n} \mid Y$ and $\cup_{n \in \mathbb{N}} V_{n}$ is dense in $\left(Y, \tau_{2} \mid Y\right)$. But $Y$ is dense in $\left(X, \tau_{2}\right)$, so $\cup_{n \in \mathbb{N}} V_{n}$ is dense in $\left(X, \tau_{2}\right)$.

Theorem 8. Let $\left(X, \tau_{1}, \tau_{2}\right)$ and $\left(Y, \sigma_{1}, \sigma_{2}\right)$ be bitopological spaces and $f: X \longrightarrow Y$ be a $\left(\tau_{i}-\sigma_{i}\right)$-continuous, $\left(\tau_{i}-\sigma_{i}\right)$-open and $\left(\tau_{j}-\sigma_{j}\right)$ continuous, $\left(\tau_{j}-\sigma_{j}\right)$-closed surjection. If $X$ is $(i, j)$-selectively ccc, then $Y$ is $(i, j)$-selectively ccc.

Proof. (For $i=1$ and $j=2)$. Let $\left(\mathscr{V}_{n}: n \in \mathbb{N}\right)$ be a sequence of $\sigma_{1}$-open families in $Y$ such that $\bigcup \mathscr{V}_{n}$ is $\sigma_{1}$-dense in $Y$. Since $f$ is $\left(\tau_{1}-\sigma_{1}\right)$ continuous and $\left(\tau_{1}-\sigma_{1}\right)$ open, for each $n \in \mathbb{N}, \mathscr{U}_{n}:=\left\{f^{\leftarrow}(V): V \in \mathscr{V}_{n}\right\}$ is $\tau_{1}$-open family in $X$ and $\bigcup \mathscr{U}_{n}$ is $\tau_{1}$-dense in $X$. Because $X$ is $(1,2)$-selectively ccc, we can pick $U_{n} \in \mathcal{U}_{n}$ with $\bigcup\left\{U_{n}: n \in \mathbb{N}\right\}$ dense in $\left(X, \tau_{2}\right)$. For the sequence $\left(V_{n}: n \in \mathbb{N}\right)$, such that $f^{\leftarrow}(V)=U_{n}$, we have $\cup_{n \in \mathbb{N}} V_{n}$ dense in $\left(Y, \sigma_{2}\right)$, since $f$ is a $\left(\tau_{2}-\sigma_{2}\right)$ continuous and $\left(\tau_{2}-\sigma_{2}\right)$ closed mapping. Thus, $\left(Y, \sigma_{1}, \sigma_{2}\right)$ is $(1,2)$ selectively ccc.

Bitopological $R$-separability has been studied by Kočinac and Özçağ in [31].

Denote by $\mathscr{D}_{1}$ and $\mathscr{D}_{2}$ the collection of all dense subsets of $\left(X, \tau_{1}\right)$ and $\left(X, \tau_{2}\right)$, respectively. We say that $X$ is $R_{\left(\tau_{i}, \tau_{j}\right)}$-separable $(i, j=1,2)$, if for each sequence 
$\left(D_{n}: n \in \mathbb{N}\right)$ of elements of $\mathscr{D}_{i}$, there are $d_{n} \in D_{n}, n \in \mathbb{N}$, such that $\left\{d_{n}: n \in \mathbb{N}\right\}$ is an element of $\mathscr{D}_{j}$, i.e., if $S_{1}\left(\mathscr{D}_{i}, \mathscr{D}_{j}\right)$ holds.

We have the following fact.

\section{Proposition 3. Every $R_{\left(\tau_{i}, \tau_{j}\right)}$-separable bispace is $(i, j)$-selectively ccc.}

Corollary 4. If the bitopological space $\left(X, \tau_{1}, \tau_{2}\right)$ has a $\tau_{i}$-closed and $\tau_{j}$-open $R_{\left(\tau_{i}, \tau_{j}\right)}$-separable subspace, then $X$ is $\left(\tau_{i}, \tau_{j}\right)$ selectively ccc.

Proof. It follows by Proposition 2 and the previous result. Let $\mathscr{K}$ denote the collection of all $k$-covers of a space $X$ (open covers such that every compact subset of $X$ is contained in a member of the cover), and let $\Omega$ denote the collection of all $\omega$-covers of $X$ (open covers such that every finite subset of $X$ is contained in a member of the cover and $X$ itself is not a member of the cover).

Example 1. If a Tychonoff space $X$ with a countable base belongs to the class $S_{1}(\mathscr{K}, \Omega)$, then ([31], Theorem 4$)$ the function bispace $\left(C(X), \tau_{p}, \tau_{k}\right)$ is $R_{\left(\tau_{k}, \tau_{p}\right)}$-separable. Therefore, this function bispace is selectively $\left(\tau_{k}, \tau_{p}\right)$-star-ccc, where $\tau_{p}$ denotes the pointwise topology, and $\tau_{k}$ denotes the compact-open topology on the set $C(X)$ of all continuous real-valued functions on a Tychonoff space $X$.

3.2. Selectively Star-ccc Bispaces. We now present a bitopological version of selectively star-ccc spaces introduced and studied in [19] (see also [32-34]).

Definition 6. A bispace $\left(X, \tau_{1}, \tau_{2}\right)$ is said to be $(i, j)$-selectively star-ccc, $i, j=1,2$, if for every sequence $\left(\mathcal{U}_{n}: n \in \mathbb{N}\right)$ of $\tau_{i}$-open weak covers and every open cover $\mathscr{C}$ of $\left(X, \tau_{j}\right)$, there is a sequence $\left(U_{n}: n \in \mathbb{N}\right)$, such that for every $n, U_{n} \in \mathcal{U}_{n}$ and St $\left(\cup_{n \in \mathbb{N}} U_{n}, \mathscr{C}\right)=X$.

The following result shows that the class of selectively star-ccc spaces is big enough.

Theorem 9. Let $\left(X, \tau_{1}, \tau_{2}\right)$ be a bispace, such that $\tau_{1} \geq \tau_{2}$. If $\left(X, \tau_{2}\right)$ is a Lindelof space, then $\left(X, \tau_{1}, \tau_{2}\right)$ is a $(1,2)$-selectively star-ccc space.

Proof. Let $\left(\mathcal{U}_{n}: n \in \mathbb{N}\right)$ be a sequence of $\tau_{1}$-open weak covers of $X$, and let $\mathscr{C}$ be an open cover of $\left(X, \tau_{2}\right)$. There exists a countable set $\mathscr{V}=\left\{C_{1}, C_{2}, \ldots\right\} \subset \mathscr{C}$, such that $X=\bigcup \mathscr{V}$. Since each $C_{i}, i \in \mathbb{N}$, is $\tau_{1}$-open, for every $n \in \mathbb{N}$, there exists $U_{n} \in \mathscr{U}_{n}$, such that $U_{n} \cap C_{n} \neq \varnothing$. It follows that $\operatorname{St}\left(\cup_{n \in \mathbb{N}} U_{n}, \mathscr{V}\right)=\bigcup \mathscr{V}=X$. This proves the theorem.

Corollary 5. The bispace $\left(\mathbb{R}, \tau_{S}, \tau\right)$, where $\mathbb{R}$ is the set of real numbers, $\tau$ is the usual metric topology, and $\tau_{S}$ is the Sorgenfrey topology, is $\left(\tau_{S}, \tau\right)$-selectively star-ccc.
Recall that a space $X$ is said to be strongly star Lindelöf, if for each open cover $\mathcal{U}$ of $X$, there is a countable set $C \subset X$, such that $\operatorname{St}(C, \mathcal{U})=X$.

Call a bispace $\left(X, \tau_{1}, \tau_{2}\right)(i, j)$-selectively 2 -star-ccc if for every $\tau_{j}$-open cover $\mathcal{U}$ of $X$ and every sequence $\left(\mathscr{V}_{n}: n \in \mathbb{N}\right)$ of $\tau_{i}$-open weak covers of $X$, there is a sequence $\left(V_{n}: n \in \mathbb{N}\right)$, such that $V_{n} \in \mathscr{V}_{n}, n \in \mathbb{N}$, and $\mathrm{St}^{2}\left(\cup_{n \in \mathbb{N}} V_{n}, \mathcal{U}\right)=X$, where $\operatorname{St}^{2}(V, \mathcal{U})=\operatorname{St}(\operatorname{St}(V, \mathcal{U}), \mathcal{U})$.

Theorem 10. Let $\left(X, \tau_{1}, \tau_{2}\right)$ be a bispace such that $\tau_{1} \geq \tau_{2}$. If $\left(X, \tau_{2}\right)$ is a strongly star Lindelöf space, then $\left(X, \tau_{1}, \tau_{2}\right)$ is an $(1,2)$-selectively 2-star-ccc space.

Proof. Let $\mathcal{U}$ be an arbitrary $\tau_{2}$-open cover of $X$ and $\left(\mathscr{V}_{n}: n \in \mathbb{N}\right)$ be a sequence of $\tau_{1}$-open weak covers of $X$. Since $\left(X, \tau_{2}\right)$ is strongly star Lindelöf, there exists a countable set $A=\left\{a_{1}, a_{2}, \ldots\right\} \subset X$, such that $\operatorname{St}(A, \mathcal{U})=X$. Therefore, $\left\{\operatorname{St}\left(a_{n}, \mathcal{U}\right): n \in \mathbb{N}\right\}$ is a $\tau_{2}$-open cover of $X$. For each $n \in \mathbb{N}$, there exists $U_{n} \in \mathcal{U}$, such that $a_{n} \in U_{n} \subset \operatorname{St}\left(a_{n}, \mathcal{U}\right)$. So, $U_{n} \subset \operatorname{St}\left(a_{n}, \mathcal{U}\right) \subset \operatorname{St}\left(U_{n}, \mathcal{U}\right)$. For every $n \in \mathbb{N}$, the set $\cup_{n \in \mathbb{N}} \cup \mathscr{V}_{n}$ is dense in $\left(X, \tau_{1}\right)$ and hence in $\left(X, \tau_{2}\right)$, so that $U_{n} \cap\left(\cup_{n \in \mathbb{N}} \mathscr{V}_{n}\right) \neq \varnothing$. This implies that for each $n \in \mathbb{N}$, there exists $V_{n} \in \mathscr{V}_{n}$, such that $U_{n} \cap V_{n} \neq \varnothing$. Therefore, for each $n \in \mathbb{N}$,

$$
\operatorname{St}\left(U_{n}, \mathcal{U}\right) \subset \operatorname{St}\left(\operatorname{St}\left(V_{n}, \mathcal{U}\right), \mathcal{U}\right)=\operatorname{St}^{2}\left(V_{n}, \mathcal{U}\right),
$$

which implies

$$
X=\operatorname{St}(A, \mathcal{U}) \subset \operatorname{St}^{2}\left(\cup_{n \in \mathbb{N}} V_{n}, \mathcal{U}\right) .
$$

This shows that $\left(X, \tau_{1}, \tau_{2}\right)$ is $(1,2)$-selectively 2 -starccc.

Theorem 11. Every $(i, j)$-selectively ccc bispace $\left(X, \tau_{1}, \tau_{2}\right)$ is $(i, j)$-selectively star-ccc.

Proof. (For $i=1, j=2$ ). Let $\mathscr{C}$ be a $\tau_{2}$-open cover of $X$ and $\left(\mathcal{U}_{n}: n \in \mathbb{N}\right.$ ) be a sequence of $\tau_{1}$-open weak covers of $X$. Since $X$ is $(1,2)$-selectively ccc, there exists a sequence $\left(U_{n}: n \in \mathbb{N}\right)$, such that for every $n, U_{n} \in \mathcal{U}_{n}$, and $M=\cup_{n \in \mathbb{N}} U_{n}$ is dense in $\left(X, \tau_{2}\right)$. So, $M$ intersects each element of $\mathscr{C}$, and thus,

$$
\operatorname{St}\left(\bigcup_{n \in \mathbb{N}} U_{n}, \mathscr{C}\right)=X,
$$

where $X$ is a $(1,2)$-selectively star-ccc space.

From Proposition 3 and Theorem 11, we have the following corollary.

Corollary 6. Every $R_{\left(\tau_{i}, \tau_{j}\right)}$-separable bispace is $(i, j)$-selectively star-ccc.

We show now that the converse in this corollary is not always true.

Example 2. Let $\left(X, \tau_{1}, \tau_{2}\right)$ be a bispace with $\tau_{1} \geq \tau_{2}$, such that $\left(X, \tau_{2}\right)$ is a compact and nonseparable space. By Theorem 9 , the bitopological space $\left(X, \tau_{1}, \tau_{2}\right)$ is $(1,2)$-selectively 
star-ccc. But it is not $R_{\left(\tau_{1}, \tau_{2}\right)}$-separable by the following fact: $R_{\left(\tau_{1}, \tau_{2}\right)}$-separability implies separability of $\left(X, \tau_{2}\right)$.

\section{Covering Properties and Relations on Bispaces}

In this section, we will investigate the behaviour and preservation of covering properties studied in this study under various relations (as $<_{\mathrm{C}}$ and $<_{\mathrm{N}}$ ) between two topologies on a bitopological space $\left(X, \tau_{1}, \tau_{2}\right)$.

In 1957, Weston [35] introduced the C-relation, while in [30], the N-relation was defined. The abovementioned relations and their combinations with the set-theoretic operation inclusion are considered in [30].

Let $\left(X, \tau_{1}, \tau_{2}\right)$ be a bitopological space. Obviously, $\tau_{2}<\tau_{1}$ implies $\mathrm{Cl}_{\tau_{1}}(A) \subset \mathrm{Cl}_{\tau_{2}}(A)$ for each $A \subset X$.

Definition 7 (see $[30,35])$. The topology $\tau_{1}$ is coupled to the topology $\tau_{2}$ on a set $X$, denoted by $\tau_{1} \mathbf{C} \tau_{2}$, if $\mathrm{Cl}_{\tau_{1}}(A) \subset \mathrm{Cl}_{\tau_{2}}(A)$ for each $A \in \tau_{1}$.

Remark 3. (see [30]). If the open sets and closed sets are equal for a topology $\tau_{1}$ on a set $X$, then $\tau_{1}$ is coupled to every topology on $X$. For example, the discrete and indiscrete topologies are coupled to each topology on $X$.

The C-relation defines a partial order $\leq$ (we use the notation $<_{\mathrm{C}}$ ) on the family of all topologies on $X$ by

$$
\tau_{1}<_{\mathrm{C}} \tau_{2} \Leftrightarrow\left(\tau_{1} \mathbf{C} \tau_{2} \wedge \tau_{1}<\tau_{2}\right) .
$$

It is easy to see that $\tau_{1}{ }_{\mathrm{C}} \tau_{2}$ implies $\left(\tau_{1} \mathrm{C} \tau_{2} \wedge \tau_{2} \mathrm{C} \tau_{1}\right)$, but the reverse implication is not correct.

Example 3. Let $X=\{a, b, c, d, e\}, \tau_{1}=\{\varnothing,\{e\},\{a, b, c, d\}, X\}$, and $\tau_{2}=\{\varnothing,\{c\},\{a, b, d, e\}, X\}$. It is clear that $\tau_{1}<_{\mathrm{C}} \tau_{2}$ and $\tau_{2}<_{\mathrm{C}} \tau_{1}$, but these topologies are not comparable by inclusion.

A set $X$ together with the topologies $\tau_{1} \mathrm{C} \tau_{2}\left(\tau_{1}<{ }_{\mathrm{C}} \tau_{2}\right)$ is denoted by $\left(X, \tau_{1} \mathrm{C} \tau_{2}\right)\left(X, \tau_{1}<{ }_{\mathrm{C}} \tau_{2}\right)$.

Now, we consider the $\mathrm{N}$-relation between two topologies on a set $X$.

Definition 8. (see [30]). A topology $\tau_{1}$ is near to a topology $\tau_{2}$ on a set $X$ (briefly $\tau_{1} \mathrm{~N} \tau_{2}$ ) if $\mathrm{Cl}_{\tau_{1}}(A) \subset \mathrm{Cl}_{\tau_{2}}(A)$ for each $A \in \tau_{2}$.

The discrete topology on $X$ is near any topology on $X$, and every topology on $X$ is near the indiscrete topology on $X$.

Similar to the C-relation, the N-relation defines a partial order on the family of all topologies on $X$ by

$$
\tau_{1}<_{\mathbf{N}} \tau_{2} \Leftrightarrow\left(\tau_{1} \mathbf{N} \tau_{2} \wedge \tau_{1}<\tau_{2}\right)
$$

We mention that the notions of coupling and nearness of topologies are independent of each other; however, we have the following implication:

$$
\tau_{1}<_{\mathbf{N}} \tau_{2} \Rightarrow \tau_{1}<_{\mathbf{C}} \tau_{2}
$$

A set $X$ together with the topologies $\tau_{1} \mathrm{~N} \tau_{2}\left(\tau_{1}<{ }_{N} \tau_{2}\right)$ is denoted by $\left(X, \tau_{1} \mathrm{~N} \tau_{2}\right)\left(\left(X, \tau_{1}<{ }_{\mathbf{N}} \tau_{2}\right)\right)$.

We omit the proof of the following proposition.

Proposition 4. Let $\left(X, \tau_{1} C \tau_{2}\right)$ be a bitopological space. If $\left(X, \tau_{1}\right)$ is almost Menger, then $\left(X, \tau_{1} C \tau_{2}\right)$ is $(1,2)$-almost Menger.

However, in the following example, we show that the reverse implication is not true in general.

Example 4. Let $X$ be the Euclidean plane $\mathbb{R}^{2}, \tau_{1}$ be the Sorgenfrey topology, and $\tau_{1}$ be the ordinary metric topology on $X$. Clearly, $\left(X, \tau_{1} \mathrm{C} \tau_{2}\right)$ is $(1,2)$-almost Menger; however, $\left(X, \tau_{1}\right)$ does not have the almost Menger property, since $\left(X, \tau_{1}\right)$ is not almost Lindelöf.

On the other hand, we have the following result.

Theorem 12. A bispace $\left(X, \tau_{1}<{ }_{\mathrm{C}} \tau_{2}\right)$ is $(1,2)$-almost Menger if and only if $\left(X, \tau_{1}\right)$ is almost Menger.

Proof. Let $\left(\mathcal{U}_{n}: n \in \mathbb{N}\right)$ be a sequence of $\tau_{1}$-open covers of $X$. Since $\left(X, \tau_{1}<_{\mathrm{C}} \tau_{2}\right)$ is $(1,2)$-almost Menger, there exists a sequence $\left(\mathscr{V}_{n}: n \in \mathbb{N}\right)$ of finite sets with $\mathscr{V}_{n} \subset \mathcal{U}_{n}$ and

$$
\cup_{n \in \mathbb{N}} \cup\left\{\mathrm{Cl}_{\tau_{2}}(V): V \in \mathscr{V}_{n}\right\}=X \text {. }
$$

By $\tau_{1}<{ }_{\mathrm{C}} \tau_{2}$, we have $\tau_{1} \mathrm{C} \tau_{2}$ and $\tau_{1}<\tau_{2}$. For $V \in \tau_{1}<\tau_{2}$, we have $\mathrm{Cl}_{\tau_{2}}(V) \subset \mathrm{Cl}_{\tau_{1}}(V)$. Therefore, $\cup_{n \in \mathbb{N}} \cup\left\{\mathrm{Cl}_{\tau_{1}}(V)\right.$ : $\left.V \in \mathscr{V}_{n}\right\}=X$ gives that $\left(X, \tau_{1}\right)$ is almost Menger.

Let $\left(\mathcal{U}_{n}: n \in \mathbb{N}\right)$ be a sequence of $\tau_{1}$-open covers of $X$. By the almost Mengerness of $\left(X, \tau_{1}\right)$, one can find finite sets $\mathscr{V}_{n} \subset \mathscr{U}_{n}, n \in \mathbb{N}$, such that $\cup_{n \in \mathbb{N}} \bigcup\left\{\mathrm{Cl}_{\tau_{1}}(V): V \in \mathscr{V}_{n}\right\}=X$. Because $\tau_{1}<\tau_{2}$, each $\mathscr{V}_{n}$ is a family of $\tau_{2}$-open sets. So, for each $n$ and each $V \in \mathscr{V}_{n}, \tau_{1}<{ }_{\mathrm{C}} \tau_{2}$ implies $\mathrm{Cl}_{\tau_{1}}(V) \subset \mathrm{Cl}_{\tau_{2}}(V)$. It follows from here that

$$
\begin{aligned}
X= & \cup \underset{n \in \mathbb{N}}{\cup} \cup\left\{\mathrm{Cl}_{\tau_{1}}(V): V \in \mathscr{V}_{n}\right\} \\
& \subset \underset{n \in \mathbb{N}}{\cup} \bigcup\left\{\mathrm{Cl}_{\tau_{2}}(V): V \in \mathscr{V}_{n}\right\}=X .
\end{aligned}
$$

Therefore, $\left(X, \tau_{1}, \tau_{2}\right)$ is $\left(\tau_{1}, \tau_{2}\right)$-almost Menger.

It should be noted that the Proposition 4 extends and generalizes the following result obtained by Özçağ and Eysen in [20].

Corollary 7. If $\left(X, \tau_{1}\right)$ is almost Menger and $\tau_{2}<\tau_{1}$, then the bitopological space $\left(X, \tau_{1}, \tau_{2}\right)$ is $(1,2)$-almost Menger.

Similar to the proof of the first part of Theorem 12, we prove the following.

Theorem 13. If $\left(X, \tau_{1}<_{\mathrm{N}} \tau_{2}\right)$ is $(1,2)$-almost Menger, then $\left(X, \tau_{1}\right)$ is almost Menger. 
Since our main aim was, as mentioned in the introduction, to investigate the behaviour of some covering properties under several functions, we turn to mappings between bitopological spaces equipped with some relations.

The classes of all $(i, j)-\theta$-continuous functions of $X$ to $Y$ are denoted by $(i, j)-\theta C(X, Y)$.

Corollary 8 (see [30]). Let $\left(X, \tau_{1}, \tau_{2}\right)$ and $\left(Y, \sigma_{1}, \sigma_{2}\right)$ be bitopological spaces. For $\left(X, \tau_{1}<{ }_{\mathrm{C}} \tau_{2}\right)$ and $\left(Y, \sigma_{1}<{ }_{\mathrm{C}} \sigma_{2}\right)$, we have

$$
(1,2)-\theta C(X, Y)=1-\theta C(X, Y) .
$$

Theorem 14. Let $f:\left(X, \tau_{1}<_{\mathrm{C}} \tau_{2}\right) \longrightarrow\left(Y, \sigma_{1}<{ }_{\mathrm{C}} \sigma_{2}\right)$ be $(1,2)$ - $\theta$-continuous mapping. If $\left(X, \tau_{1}<_{\mathrm{C}} \tau_{2}\right)$ is $(1,2)$-almost Menger, then

Proof. Let $\left(X, \tau_{1}<{ }_{\mathrm{C}} \tau_{2}\right)$ be an $(1,2)$-almost Menger bitopological space. By Theorem $12,\left(X, \tau_{1}\right)$ is almost Menger, and the mapping $f_{i}:\left(X, \tau_{1}\right) \longrightarrow\left(Y, \sigma_{1}\right)$ is $\theta$-continuous by Corollary 8. Since $\theta$-continuous image of an almost Menger space is almost Menger ([36], Theorem 2.1), then $\left(Y, \sigma_{1}\right)$ is almost Menger. Thus, $\left(Y, \sigma_{1}<{ }_{\mathrm{C}} \sigma_{2}\right)$ is $(1,2)$-almost Menger by Theorem 12 .

Corollary 9 (see [30]). The following conditions are satisfied for a bitopological space $\left(X, \tau_{1} C \tau_{2}\right)$.

(1) If $\left(X, \tau_{1}, \tau_{2}\right)$ is $(1,2)$-regular, then $\left(X, \tau_{1}, \tau_{2}\right)$ is 1 regular

Moreover, for a bitopological space $\left(X, \tau_{1}<{ }_{\mathrm{C}} \tau_{2}\right)$, we have

(2) $\left(X, \tau_{1}, \tau_{2}\right)$ is $(1,2)$-regular $\Leftrightarrow\left(X, \tau_{1}, \tau_{2}\right)$ is 1-regular

Theorem 15. Let $\left(X, \tau_{1}<{ }_{\mathrm{C}} \tau_{2}\right)$ be $(1,2)$-regular and $(1,2)$-almost Menger bitopological space. If $f:\left(X, \tau_{1}, \tau_{2}\right) \longrightarrow$ $\left(Y, \sigma_{1}, \sigma_{2}\right)$ is 1-continuous mapping, then $\left(Y, \sigma_{1}<{ }_{\mathrm{C}} \sigma_{2}\right)$ is (1,2)-almost Menger.

Proof. Let $\left(X, \tau_{1}<{ }_{\mathrm{C}} \tau_{2}\right)$ be $(1,2)$-almost Menger and $(1,2)$-regular bitopological space, then $\left(X, \tau_{1}\right)$ is almost Menger by Theorem 12 and $\left(X, \tau_{1}\right)$ is regular by Corollary 9 ; thus, we obtain that $\left(X, \tau_{1}\right)$ is Menger ([10], Theorem 2.1). Since $f:\left(X, \tau_{1}\right) \longrightarrow\left(Y, \sigma_{1}\right)$ is continuous and continuous image of a Menger space is Menger, then $\left(Y, \sigma_{1}\right)$ is Menger and $\left(Y, \sigma_{1}\right)$ is almost Menger. By using Theorem 12, $\left(Y, \sigma_{1}<{ }_{\mathrm{C}} \sigma_{2}\right)$ is $(1,2)$-almost Menger.

Proposition 5. If $f:\left(X, \tau_{1} C \tau_{2}\right) \longrightarrow\left(Y, \sigma_{1}, \sigma_{2}\right)$ is $(1,2)$ -strongly $\theta$-continuous mapping, then $f$ is 1-strongly $\theta$-continuous.

Proof. Let $f:\left(X, \tau_{1} \mathrm{C} \tau_{2}\right) \longrightarrow\left(Y, \sigma_{1}, \sigma_{2}\right)$ be $(1,2)$-strongly $\theta$-continuous mapping. Let $x \in X$ and $V$ be $\sigma_{1}$-open set in $Y$ containing $f(x)$. Since $f$ is $(1,2)$-strongly $\theta$-continuous mapping, there is a $\tau_{1}$-open set $U$ in $X$ containing $x$, such that $f\left(\mathrm{Cl}_{\tau_{2}}(U)\right) \subseteq V$. Since $\tau_{1} \mathrm{C} \tau_{2}$, we have $f\left(\mathrm{Cl}_{\tau_{1}}(U)\right) \subseteq$ $f\left(\mathrm{Cl}_{\tau_{2}}(U)\right) \subseteq V$; thus, $f$ is 1-strongly $\theta$-continuous.
Proposition 6. $f:\left(X, \tau_{1}<{ }_{\mathrm{C}} \tau_{2}\right) \longrightarrow\left(Y, \sigma_{1}, \sigma_{2}\right)$ is $(1,2)$ -strongly $\theta$-continuous mapping if and only if $f$ is 1-strongly $\theta$-continuous.

Proof. Immediate.

Corollary 10. Let $f:\left(X, \tau_{1}<_{\mathrm{C}} \tau_{2}\right) \longrightarrow\left(Y, \sigma_{1}, \sigma_{1}\right)$ be $(i, j)$-strongly $\theta$-continuous mapping. If $\left(X, \tau_{1}<{ }_{\mathrm{C}} \tau_{2}\right)$ is $(1,2)$-almost Menger, then $\left(Y, \sigma_{1}\right)$ is Menger.

Proof. If $\left(X, \tau_{1}<{ }_{\mathrm{C}} \tau_{2}\right)$ is $(i, j)$-almost Menger, then by Proposition 4, $\left(X, \tau_{1}\right)$ is almost Menger. Since $f:\left(X, \tau_{1}\right) \longrightarrow\left(Y, \sigma_{1}\right)$ is strongly $\theta$-continuous by the Proposition 5, we obtain that $\left(Y, \sigma_{1}\right)$ is Menger by ([36], Theorem 2.7).

\subsection{More on Relations and Covering Properties}

Example 5. Lindelöfness is not preserved by the order relation $<_{\mathrm{C}}$.

(1) Let $\left(X, \tau_{1}, \tau_{2}\right)$ be the plane $\mathbb{R}^{2}$ equipped with the following two topologies: $\tau_{1}$ is the usual Euclidean topology, and $\tau_{2}$ is the deleted radius topology ([37], example 77). Then, $\tau_{1}<\tau_{2}$, and for any open set $U \in \tau_{1}, \mathrm{Cl}_{\tau_{1}}(U)=\mathrm{Cl}_{\tau_{2}}(U)$, i.e., $\tau_{1}<_{\mathrm{C}} \tau_{2} .\left(X, \tau_{1}\right)$ is a Lindelöf spaces, while $\left(X, \tau_{2}\right)$ is not ([37], example 77).

(2) Another such example is the set $X=\left\{(x, y) \in \mathbb{R}^{2}\right.$ : $y \geq 0\}$ with the Euclidean topology $\left(\tau_{1}\right)$ and the Niemytzki plane topology $\left(\tau_{2}\right)$

Example 6. $\sigma$-compactness is not preserved by the order relation $<_{\mathrm{C}}$

Let $\left(X, \tau_{1}, \tau_{2}\right)$ be the plane $\mathbb{R}^{2}$ equipped with the following two topologies: $\tau_{1}$ is the usual Euclidean topology, and $\tau_{2}$ is the deleted radius topology ([37], example 77). It is clear that $\tau_{1}{ }_{\mathrm{C}} \tau_{2} .\left(X, \tau_{1}\right)$ is $\sigma$-compact, but $\left(X, \tau_{2}\right)$ is not $\sigma$-compact.

Remark 4. Being $\sigma$-compact, the space $\left(X, \tau_{1}\right)$ in the previous example is Alster [8]. However, $\left(X, \tau_{2}\right)$ is not Alster because it is not Menger (Alster spaces are Hurewicz; hence, Menger in all finite powers).

Example 7. Mengerness is not preserved by the order relation $<_{\mathrm{N}}$.

Let $X=\left\{(x, y) \in \mathbb{R}^{2}: y \geq 0\right\}$. Let $Y=\left\{(x, y) \in \mathbb{R}^{2}\right.$ : $y>0\}$. Let $\tau_{1}$ be the usual metric topology on $X$, and $\tau_{2}$, the half-disk topology ([37], example 78), is defined as follows: any point $p \in Y$ has the Euclidean local base, while the point $p=(x, 0) \in X \backslash Y$ has basic neighbourhoods of the form $B(p, \varepsilon) \cap Y) \cup\{p\}$. Then, $\tau_{1}<\tau_{2}$, and for any $\tau_{2}$-open set $U$, $\mathrm{Cl}_{\tau_{2}}(U)=\mathrm{Cl}_{\tau_{1}}(U)$, so that $\tau_{1}<_{\mathrm{N}} \tau_{2}$. The space $\left(X, \tau_{1}\right)$ is Menger, while $\left(X, \tau_{2}\right)$ is not Menger because it is not Lindelöf. 
TABLE 1: The preservation of some covering properties under various relations.

\begin{tabular}{|c|c|c|c|c|c|}
\hline & Property & $\tau_{1} \mathrm{C} \tau_{2}$ & $\tau_{1} \mathrm{~N} \tau_{2}$ & $\tau_{1} \leq_{C} \tau_{2}$ & $\tau_{1} \leq{ }_{N} \tau_{2}$ \\
\hline 1 & $\tau_{1} \mathrm{~L}$ & & & $\tau_{2} \mathrm{~L}$ & \\
\hline 2 & $\tau_{1} \sigma-\mathrm{com}$ & & & $\tau_{2} \sigma-\operatorname{com}$ & \\
\hline 3 & $\tau_{1} \mathrm{M}$ & & & $\tau_{2} \mathrm{M}$ & \\
\hline 4 & $\tau_{2} \mathrm{aM}$ & & & & $\tau_{1} \mathrm{aM}$ \\
\hline 5 & $\tau_{2} \mathrm{wM}$ & & & & $\tau_{1} \mathrm{wM}$ \\
\hline 6 & $\tau_{1} \mathrm{H}$ & & & $\tau_{2} \mathrm{H}$ & \\
\hline 7 & $\tau_{2} \mathrm{R}$ & & $\tau_{1} \mathrm{R}$ & & \\
\hline 8 & $\tau_{1}$ Alster & & & $\tau_{2}$ Alster & \\
\hline 9 & $\tau_{2}$ sel ccc & & & $\tau_{1}$ sel ccc & \\
\hline
\end{tabular}

Example 8. Hurewicz property is not preserved by the order relation $<\mathrm{C}$.

Let $\left(X, \tau_{1}, \tau_{2}\right)$ be the plane $\mathbb{R}^{2}$ equipped with the following two topologies: $\tau_{1}$ is the usual Euclidean topology, and $\tau_{2}$ is the deleted diameter topology ([37], Example 77). It is clear that $\tau_{1}{ }_{{ }_{C}} \tau_{2} .\left(X, \tau_{1}\right)$ is the Hurewicz, but $\left(X, \tau_{2}\right)$ is not Hurewicz because it is not Lindelöf.

Another such example is the closed upper half-plane with the Euclidean topology $\tau_{1}$ and the half-disc topology $\tau_{2}$ ([37], example 78).

Example 9. Let $X$ be any uncountable scattered compact space (for instance, the product $\left[0, \omega_{1}\right] \times[0, \omega]$ of ordinal spaces) with the topology $\tau_{2}$, and let $\tau_{1}$ be the discrete topology on $X$. Then, $\tau_{1} N \tau_{2},\left(X, \tau_{2}\right)$ is a Rothberger space, and $\left(X, \tau_{1}\right)$ is not Rothberger.

Theorem 16. If $\tau_{1}<{ }_{\mathrm{N}} \tau_{2}$ and $\left(X, \tau_{2}\right)$ is almost Menger, then $\left(X, \tau_{1}\right)$ is almost Menger.

Proof. Let $\left(\mathscr{U}_{n}: n \in \mathbb{N}\right)$ be a sequence of open covers of $\left(X, \tau_{1}\right)$. As $\tau_{1}<\tau_{2}$, it is a sequence of open covers of $\left(X, \tau_{2}\right)$. By assumption, there are finite sets $\mathscr{V}_{n} \subset \mathscr{U}_{n}, n \in \mathbb{N}$, so that $X=\cup_{n \in \mathbb{N}} \mathrm{Cl}_{\tau_{2}}\left(\bigcup \mathscr{V}_{n}\right)$. From $\tau_{1}<_{\mathrm{N}} \tau_{2}$ it follows $\mathrm{Cl}_{\tau_{2}}\left(\bigcup \mathscr{V}_{n}\right)=\mathrm{Cl}_{\tau_{1}}\left(\bigcup \mathscr{V}_{n}\right)$ for each $n \in \mathbb{N}$, which means that $\left(X, \tau_{1}\right)$ is almost Menger.

Similarly, one obtains.

Theorem 17. If $\tau_{1}<_{\mathrm{N}} \tau_{2}$ and $\left(X, \tau_{2}\right)$ is weakly Menger, then $\left(X, \tau_{1}\right)$ is weakly Menger.

Theorem 18. Let $\left(X, \tau_{1}, \tau_{2}\right)$ be a bitopological space. If $\tau_{1}<_{\mathrm{C}} \tau_{2}$ and $\left(X, \tau_{2}\right)$ is selectively $c c c$, then $\left(X, \tau_{1}\right)$ is selectively $c c c$.

Proof. Let $\left(\mathscr{U}_{n}: n \in \mathbb{N}\right)$ be a sequence of $\tau_{1}$-open weak cover of $X$. Then, $\bigcup \mathscr{U}_{n} \in \tau_{1}, \tau_{1}<{ }_{\mathrm{C}} \tau_{2}$ and $\mathrm{Cl}_{\tau_{1}}\left(\bigcup \mathscr{U}_{n}\right)=X$, $n \in \mathbb{N}$, imply that for each $n, \mathscr{U}_{n} \in \tau_{2}$, and $\mathrm{Cl}_{\tau_{2}}\left(\bigcup \mathcal{U}_{n}\right)=X$. As $\left(X, \tau_{2}\right)$ is selectively ccc, there are $U_{n} \in \mathscr{U}_{n}, n \in \mathbb{N}$, such that $\mathrm{Cl}_{\tau_{2}}\left(\cup_{n \in \mathbb{N}} U_{n}\right)=X$. From $\tau_{1}<\tau_{2}$, it follows $\mathrm{Cl}_{\tau_{1}}\left(\cup_{n \in \mathbb{N}} U_{n}\right)^{C \mathrm{C}_{\tau_{2}}}\left(\cup_{n \in \mathbb{N}} U_{n}\right)=\dot{X}$, which means that $\left(X, \tau_{1}\right)$ is selectively ccc.
Theorem 19. Let $\left(X, \tau_{1}, \tau_{2}\right)$ be a bitopological space. If $\tau_{1}<{ }_{C} \tau_{2}$ and $\left(X, \tau_{2}\right)$ is selectively star-ccc, then $\left(X, \tau_{1}\right)$ is selectively star-ccc.

Proof. Let $\left(\mathcal{U}_{n}\right)_{n \in \mathbb{N}}$ be a sequence of open weak covers of $\left(X, \tau_{1}\right)$ and $\mathscr{U}$ a $\tau_{1}$-open cover of $X$. Since $\bigcup \mathcal{U}_{n} \in \tau_{1}, n \in \mathbb{N}$, and $\tau_{1}<{ }_{\mathrm{C}} \tau_{2}$, we have $\mathrm{Cl}_{\tau_{1}}\left(\bigcup \mathcal{U}_{n}\right) \subset \mathrm{Cl}_{\tau_{2}}\left(\bigcup \mathcal{U}_{n}\right)=X$. Also, $\mathcal{U}$ is a $\tau_{2}$-open cover of $X$. Using the fact that $\left(X, \tau_{2}\right)$ is selectively star-ccc, we find $U_{n} \in \mathscr{U}_{n}, n \in \mathbb{N}$, such that $\operatorname{St}\left(\cup_{n \in \mathbb{N}} U_{n}, \mathscr{U}\right)=X$, i.e., $\left(X, \tau_{1}\right)$ is selectively star-ccc.

Table 1 summarizes the above results. Empty fields in the table may serve as open questions.

\section{Conclusion}

This study is a continuation and extension of our research in the field of selective covering properties in bitopological spaces. Complementing some earlier studies on almost Menger and almost Rothberger bitopological spaces, we investigated preservation of these properties under various classes of mappings between bitopological spaces. Next, we introduced and studied in detail the selective bitopological versions of the topological ccc property called selectively ccc and selectively star-ccc property. Relations of these bitopological properties with other known properties are established. In the last part of the study, we discussed (non) preservation of a number of topological and bitopological covering properties under known relations between the two topologies. We believe that a natural continuation of our study may be findings of relations of the properties studied in this study with game theory, function spaces, and hyperspaces.

\section{Data Availability}

The data used to support the findings of this study are included within the article as references and are available from the corresponding author upon request.

\section{Conflicts of Interest}

The authors declare that there are no conflicts of interest.

\section{Acknowledgments}

The authors are grateful to the referees for a number of valuable suggestions which led to improvements on several places in the study.

\section{References}

[1] R. Engelking, General Topology Sigma Series in Pure Mathematics, Heldermann, Berlin, Germany, 2 edition, 1989.

[2] J. C. Kelly, "Bitopological spaces," Proceedings of the London Mathematical Society, vol. 3, no. 1, pp. 71-89, 1963.

[3] M. Scheepers, "Combinatorics of open covers I: Ramsey theory," Topology and its Applications, vol. 69, no. 1, pp. 31-62, 1996.

[4] K. Menger, "Einige Überdeckungssätze der Punktmengenlehre," Sitzungsberichte Abt. 2a, Mathematik, Astronomie, Physik, Meteorologie und Mechanik (Wiener Akademie, Wien), vol. 133, pp. 421-444, 1924. 
[5] F. Rothberger, "Eine Verschärfung der Eigenschaft C," Fundamenta Mathematicae, vol. 30, pp. 50-55, 1938.

[6] P. Daniels, "Pixley-Roy spaces over subsets of the reals," Topology and its Applications, vol. 29, no. 1, pp. 93-106, 1988.

[7] L. Babinkostova, B. A. Pansera, and M. Scheepers, "Weak covering properties and infinite games," Topology and its Applications, vol. 159, no. 17, pp. 3644-3657, 2012.

[8] L. Babinkostova, B. A. Pansera, and M. Scheepers, "Weak covering properties and selection principles," Topology and its Applications, vol. 160, no. 18, pp. 2251-2271, 2013.

[9] L. D. R. Kočinac, "Star-Menger and related spaces II," Filomat, vol. 13, pp. 129-140, 1999.

[10] D. Kocev, "Almost Menger and related spaces," Matematički Vesnik, vol. 61, no. 2, pp. 173-180, 2009.

[11] W. Hurewicz, "Uber die Verallgemeinerung des Borelschen theorems," Mathematische Zeitschrift, vol. 24, pp. 401-425, 1925.

[12] M. Sakai and M. Scheepers, The Combinatorics of Open Covers Recent Progress in General Topology III, K. P. Hart, J. van Mill, and P. Simon, Eds., Atlantis Press, Paris, France, 2014.

[13] L. F. Aurichi, "Selectively ccc spaces," Topology and its Applications, vol. 160, no. 18, pp. 2243-2250, 2013.

[14] M. Scheepers, "Combinatorics of open covers (V): Pixley-Roy spaces of sets of reals, and $\omega$-covers," Topology and its Applications, vol. 102, no. 1, pp. 13-31, 2000.

[15] M. Scheepers, "Combinatorics of open covers (VI). Selectors for sequences of dense sets," Quaestiones Mathematicae, vol. 22, pp. 109-130, 1999.

[16] M. Bonanzinga, F. Cammaroto, B. A. Pansera, and B. Tsaban, "Diagonalizations of dense families," Topology and its Applications, vol. 165, pp. 12-25, 2014.

[17] L. D. R. Kočinac, "Star-Menger and related spaces," Publicationes Mathematicae Debrecen, vol. 55, no. 3-4, pp. 421-431, 1999.

[18] P. Bal and S. Bhowmik, "Star-selection principles: another new direction," Journal of the Indian Mathematical Society, vol. 84, no. 1-2, pp. 1-6, 2017.

[19] P. Bal and L. D. R. Kočinac, "On selectively star-ccc spaces," Topology and its Applications, vol. 37, no. 8, pp. 1121-1153, 2020.

[20] S. Özçă̆ and A. E. Eysen, "Almost Menger property in bitopological spaces," Ukrainian Mathematical Journal, vol. 68, no. 6, pp. 950-958, 2016.

[21] A. E. Eysen and S. Özçağ, "Weaker forms of the Menger property in bitopological spaces," Quaestiones Mathematicae, vol. 41, no. 7, pp. 877-888, 2018.

[22] A. E. Eysen and S. Özçağ, "Investigations on the weak versions of the Alster property in bitopological spaces and selection principles," Filomat, vol. 33, no. 14, pp. 4561-4571, 2019.

[23] L. D. R. Kočinac and S. Özçağ, "Bitopological spaces and selection principles," in Proceedings of the International Conference on Topology and its Applications, Cambridge Scientific Publishers, Islamabad, Pakistan, July 2011.

[24] D. Lyakhovets and A. V. Osipov, "Selection principles and games in bitopological function spaces," Filomat, vol. 33, no. 14, pp. 4535-4540, 2019.

[25] A. V. Osipov and S. Özçağ, "Variations of selective separability and tightness in function spaces with set-open topologies," Topology and its Applications, vol. 217, pp. 38-50, 2017.

[26] S. Bose and D. Sinha, "Almost open, almost closed, $\theta$ continuous, almost quasi compact mappings in bitopological spaces," Bulletin of the Calcutta Mathematical Society, vol. 73, pp. 345-354, 1981.
[27] G. K. Banerjee, "On pairwise almost strongly $\theta$ continuous mappings," Bulletin of the Calcutta Mathematical Society, vol. 79, pp. 314-320, 1987.

[28] S. N. Maheshwari and R. Prasad, "Semi open sets and semi continuous function in bitopological spaces," Mathematical Notes, vol. 26, pp. 29-37, 1977.

[29] F. H. Khedr and A. M. Alshibani, "On pairwise super continuous mappings in bitopological space," Internatinal Journal of Mathematics and Mathematical Sciences, vol. 14, no. 4, pp. 715-722, 1991.

[30] B. Dvalishvili, "Bitopological spaces: theory, relations with generalized algebraic structures and Applications," North Holland Mathematics Studies, Elsevier, Amsterdam, Netherlands, 2005.

[31] L. D. R. Kočinac and S. Özçağ, "Versions of separability in bitopological spaces," Topology and its Applications, vol. 158, no. 12, pp. 1471-1477, 2011.

[32] Y.-K. Song and W.-F. Xuan, "A note on selectively star-ccc spaces," Topology and its Applications, vol. 263, pp. 343-349, 2019.

[33] Y.-K. Song and W.-F. Xuan, "More on selectively star-ccc spaces," Topology and its Applications, vol. 268, Article ID 106905, 2019.

[34] W.-F. Xuan and Y.-K. Song, "A study of selectively star-ccc spaces," Topology and its Applications, vol. 273, Article ID 107103, 2020.

[35] J. D. Weston, "On the comparison of topologies," Journal of London Mathematical Society, vol. 32, no. 3, pp. 342-354, 1957.

[36] L. D. R. Kočinac, "A note on mappings and almost Menger and related spaces," University Thought: Publications in Natural Sciences, vol. 6, no. 1, pp. 67-70, 2016.

[37] L. A. Steen and J. A. Seebach Jr., Counterexamples in Topology, Dover Publications Inc, Garden city, NY, USA, 1996. 